\title{
Introducing the BDA Theatre 2022
}

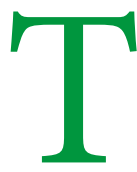

he British Dental Conference and Dentistry Show Birmingham will be returning to its live event format, offering a myriad of benefits for all members of the dental team. Among the exciting features making this a highly anticipated event will be the BDA Theatre - programmed and presented by the British Dental Association. So, what can you expect from this year's BDA Theatre?

\section{Riaz Yar}

'Occlusion - it's an emergency!'

Dr Yar will consider the supra occluded crown and its associated risks, discussing how clinicians can manage these types of cases with a simplified, conformative occlusal protocol. He will look at some examples of emergency occlusal scenarios and explore how the GDP can deal with urgent situations for the best short- and long-term outcomes.

\section{Lochana Nanayakkara}

'Bleeding hearts - how can we make a positive impact?'

The ageing population presents various challenges for the entire healthcare sector. Dr Nanayakkara will discuss patients with bleeding disorders, heart disease or a history of endocarditis, talking about how these impact quality of life as well as how dental professionals may provide support.

\section{Len D'Cruz}

'Fearful of litigation? How to stop worrying and start enjoying dentistry again'

Dr D'Cruz will be offering practical advice on how dentists can mitigate the risk of complaints being made against them. $\mathrm{He}$ will cover potential triggers for complaints and highlight the importance of good communication with patients to build trust and enhance the dentist-patient relationship. Dr D'Cruz will also reflect on the downward trend of the GDC and clinical negligence claims in general.

\section{Amit Patel}

'Unravelling the new perio classification system' As a Specialist Periodontist, Dr Patel will be exploring the new periodontal classification system, potential challenges it presents and solutions peers can utilise in practice. The session will cover non-surgical periodontal therapy options, as well as emphasising the patient's roles and responsibilities in arresting disease progression.

\section{Colin McGuirk}

'Modern dental materials and their use to deliver more predictable dentistry: where to use, when to use and what to do with them' Case selection is an essential part of fully utilising the benefits afforded by various dental materials on the market today. Dr McGuirk will discuss how to make the most of material advantages for highly aesthetic and durable restorations, especially in compromised situations.

\section{Oliver Bailey}

'Sub-gingival posterior composites, how confident are you? A step-by-step approach for the GDP' Dr Bailey will explore a simple and predictable approach to sub-gingival composites. This is an area that recent research by Newcastle University and the BDA found most GDPs lack confidence in, so it's a well-timed session that delegates will be no doubt be interested in attending!

\section{Urshla Devalia}

'How we can improve the oral health of vulnerable children and young people' Identifying and supporting vulnerable children and young people can be a difficult yet vital part of the modern dental professional's remit. Dr Devalia will be offering expert guidance to help delegates improve their approach to these difficult situations.

\section{Wendy Newman, Maeve McLernon and Deborah Gray}

'BDA CDS Group: Special care tips for everyday dentistry'

Sharing their specialist skills and extensive experience, Drs Newman, McLernon and Gray will how dentists can improve their care for patients with specialist needs and unique cases. Topics covered will include dental issues in spinal cord injuries, dysphagia in dentistry and domiciliary care.

\section{Graham Smith}

'The urgent two-week rule: suspicious lesions of the head and neck, what not to miss'

Early diagnosis and treatment are crucial for patient survival of head and neck cancer. Dr

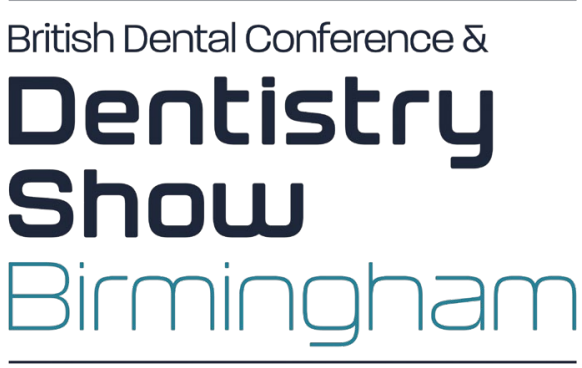

13-14 May 2022 | NEC | Hall 5

Smith will talk about which patients should be referred on the two-week rule, how they should be managed and why the dental professional's role is so important for both the monitoring and prevention of head and neck cancer.

\section{Finlay Sutton}

'The 7 top techniques for making superb complete and partial dentures'

Dr Sutton is well-known for his expertise within the prosthodontic field. He will be joining the BDA Theatre line-up to present a step-by-step clinical technique for the delivery of highly functional and aesthetic dentures, providing a wealth of practical advice and guidance for delegates to make the most of.

\section{Sarra Jawad}

'How complex is complex?'

Join Dr Jawad to reflect upon cases that are particularly difficult to treat or unique in their treatment needs. With examples of cases referred by general dental practices, delegates will gain insight into how complex cases can be managed effectively.

Throughout all of this, attendees will have the opportunity to engage with both speakers and peers from within and outside the BDA community. There will also be plenty of opportunity to interact with colleagues from across the profession and join conversations about cutting-edge products, techniques and materials.

The British Dental Conference and Dentistry Show Birmingham will be held on Friday 13 and Saturday 14 May 2022, Birmingham NEC, co-located with DTS. For more information, visit birmingham. dentistryshow.co.uk, or email dentistry@ closerstillmedia.com. 